\title{
CHARACTERIZATION OF SOCIABILITY ON ONLINE SOCIAL NETWORKS IN BRAZILIAN ELDERLY' PERSPECTIVE: A CASE STUDY ON FACEBOOK
}

\author{
Thiago Alexandre de Souza Silva, Natália Moreira de Paula, Glívia Angélica Rodrigues Barbosa, \\ Ismael Santana Silva and Flávio Roberto dos Santos Coutinho \\ Centro Federal de Educação Tecnológica de Minas Gerais \\ Belo Horizonte, Brazil
}

\begin{abstract}
This work aimed to characterize how online social networks support the sociability among elderly members of Brazil and to discuss the impact of this proposed sociability in the social interaction of these users. To achieve this goal, we conducted a case study on Facebook, which aimed to evaluate the sociability on this online social network in the perspectives of experts in HCI and elderly users view point. The results showed that the interface and interaction model of Facebook reflects on the experience of use and sociability of this members' group. As contributions, we present insights about the design and evaluation of social software more focused on the sociability of elderly users.
\end{abstract}

\section{KEYWORDS}

Online Social Networks, Elderly People, User Experience, Sociability, Facebook, Interface Evaluation

\section{INTRODUCTION}

An aging population brings challenges, such as keeping elderly people socially included, once the physical limitations due to age and changes in behavior may contribute to their social isolation (Braun, 2013). To minimize this isolation, there are initiatives that make use of Information and Communication Technologies (ICTs) to promote the social inclusion of this group of people (Chen and Schulz, 2016). For example, online social networks have features that facilitate the socialization of their users regardless of distance and time. Thus, when appropriate, these online networks can assist elderly people in interacting with their family, friends, other stakeholders, and keep them up-to-date on everyday issues. Thus, online social networks have resources that can help combat the social isolation of elderly people (Chen and Schulz, 2016).

However, the social inclusion of elderly population through online social networks may face difficulties (Chen and Schulz, 2016), once aspects that influence the use experience on such networks (e.g., communicability, usability, accessibility and sociability) may not be adequate to the specific needs of this group of users (Chen and Schulz, 2016). In other words, to enhance the quality of social interaction (i.e., sociability (Souza and Preece, 2004)) on social software to elderly people, it is necessary to adapt this kind of system in an inclusive way, taking into account the needs, constraints and visions of these users in relation to these platforms (Chen and Schulz, 2016) (Chen and Schulz, 2016). Therefore, it is necessary to conduct studies to investigate how online social networks have been supporting the interactions of elderly users, so that the demands of this group can be exposed and improvements can be discussed for that these networks become appropriate tools for the socialization of this group platforms (Chen and Schulz, 2016).

Motivated by this demand, the aim of this work is to evaluate and to characterize the sociability on online social networks from the perspective of Brazilian elderly users, through a case study on Facebook, the most used online social network in Brazil and in the world. To achieve the proposed goal, we investigated the following research question: "Does the Facebook interface and interaction model support social interaction among elderly users?" 
The methodology to answer this question consisted in a three-step approach. Initially, we performed an evaluation from the perspective of Human-Computer Interaction (HCI) experts to analyze if the resources offered by Facebook are adequate to user experience (UX) (Nielsen and Budiu, 2015) and sociability guidelines (Souza and Preece, 2004). Subsequently, an evaluation from the perspective of elderly users was conducted with the purpose of analyzing the experience of use during the social interaction mediated by this system. Finally, we triangulated the evaluations' results and we discussed the impact of Facebook on the sociability of elderly people.

The main results allow a reflection about (1) how online social networks have been supporting the social interaction of elderly people and (2) the needs of this public in relation to this type of service. Thus, this research contributes to the HCI community may reflect about the importance of designing online social networks that enhance the satisfactory experience of elderly users, so as to stimulate the social inclusion of these users through this type of technological service (Chen and Schulz, 2016). In a complementary way, this work also reinforces the importance of sociability as a strategy to improve the user experience and social interaction on social software. Therefore, the findings presented here provide insights that are useful for designing and evaluating online social networks more focused on the sociability of elderly people in the future.

\section{RELATED WORKS}

In the literature, it is possible to find works such as those performed by Braun, 2013 and Bell et al., 2013, which aimed to characterize elderly people's perceptions in relation to online social networks, as well as the factors that encourage and discourage this group of users to use this type of online service. The research conducted by Bell et al., 2013 analyzed the opinions and perceptions of 142 North American elders and the work of Braun, 2013 was attended by 124 elderly people from Europe. Although these works were conducted in different regions, the results achieved were convergent and indicated that factors such as perception of utility and trust in the network contribute to elderly people begin to use online social networks. On the other hand, factors such as difficulties in interaction as well as social pressure negatively affect the use of this service by this public (Braun, 2013) (Bell et al., 2013). Thus, the papers discuss and present the demand for new research that: (1) characterize the needs of elderly people in relation to social software and (2) discuss how online social networks have been supporting the interaction of this group of users currently. According these researchers, from these investigate lines, it will be possible to implement improvements in these virtual environments directed to this public (Braun, 2013) (Bell et al., 2013).

Furthermore, Chen and Schulz, 2016 conducted a systematic review in the literature to characterize the effects that ICTs have on reducing the social isolation of elderly people. The review contemplated full articles related to the theme, published in English between 2002 and 2015. The proposed characterization made it possible to explain that, although they stand out as a valid alternative, ICTs do not always support the digital and social inclusion of old people adequately. The study also showed that barriers to this inclusion are associated with both social issues (e.g., resistance of elderly people to adopting technologies, family pressure), and difficulties with technology (e.g., complex functionalities and interfaces) (Chen and Schulz, 2016).

Given the results of this research, Chen and Schulz, 2016 suggest new studies: (1) to investigate and to discuss why current ICTs still present barriers for the interaction of elderly people, despite of their potential to the inclusion for this group of users, (2) to characterize which types of technologies can contribute to combat the social isolation of this group, and (3) to provide insights that contribute to the design and evaluation of ICTs that are more geared towards the social inclusion of elderly users (Chen and Schulz, 2016).

In this sense, the present work differs and complements previous research, since it seeks to evaluate and characterize the experience of use and sociability on online social networks from the perspective of Brazilian elderly users, through a case study on Facebook. Thus, the results of this work can contribute to the design of online social networks more focused on the use experience and sociability of elderly users (Braun, 2013). 


\section{ELDERLY SOCIABILITY ON FACEBOOK THROUGH EXPERTS' PERSPECTIVE}

In the first step of our research, we performed an evaluation in the perspective of HCI experts to analyze whether the features offered by Facebook are adequate to UX guidelines (Nielsen and Budiu, 2015) and also sociability guidelines (Souza and Preece, 2004) available in literature, considering the profile of elderly users. We performed the proposed evaluation using the Heuristic Evaluation (HE) (Nielsen, 1994). The HE is an inspection method to evaluate interfaces and it is conducted by HCI experts, without the participation of users. During the evaluation following the steps of this method, the expert inspects the interface looking for potential problems which violate one or more guidelines (also called heuristics) that describe principles of quality of use (e.g., usability, UX, sociability). Subsequently, the identified problems are categorized according to their severity (i.e., catastrophic, large, small, cosmetic) taking into account the potential impact generated in user interaction (Nielsen, 1994).

As the set of guidelines to guide the HE can be modified or adapted according to the purpose of the inspection (Nielsen, 1994), to evaluate the appropriateness of Facebook's resources to the principles of UX and sociability in the context of interaction of elderly users, we conducted the HE using the UX guidelines presented by Nielsen and Budiu, 2015 and the sociability guidelines presented by Souza and Preece, 2004. These guidelines were adopted since user experience impacts on the quality of social interaction (i.e., sociability) mediated systems (Nielsen and Budiu, 2015)(Souza and Preece, 2004).

The guidelines of UX (Nielsen and Budiu, 2015) used were: UX1 - Contextualization for the user and adequacy to the resources; UX2 - Error handling and user assistance; UX3 - Clear, immediate and informative feedback; UX4 - Explain the interaction paths; UX5 - Alternatives in text; UX6 - Adaptable; UX7 - Perceptible; UX8 - Responsive design; UX9 - Navigable; UX10 - Readable; UX11 - Predictability and consistency; UX12 - Visibility and easy access to all existing information; UX13 - Minimizing user memory load; UX14 - Easy access to resources; UX15 - Help and documentation; UX16 - Good use of screen space; and UX17 - Encouraging interaction. On the other hand, the sociability guidelines (Souza and Preece, 2004) used were: S1 - Allowing people to develop their own identities online; S2 - Encourage empathy and trust among members; S3 - Encourage reciprocity; S4 - Maintain discussions on evidence and topics; S5 - Maintain different communication and interaction options; and S6 - Ensure protection and privacy.

We conducted the evaluation in the first half of January 2018 by two evaluators, with at least eight years' experience in the application of $\mathrm{HE}$ and by an Occupational Therapist specializing in the rehabilitation and inclusion of elderly people, with more than five years of experience in the area. The participation of this Occupational Therapist specialist contributed to that the HCI specialists could justify why the potential problems identified during the evaluation on Facebook could affect the quality of the social interaction of elderly users on this system.

During the evaluation, the experts considered eight distinct interaction scenarios on Facebook: (1) Create profile and provide basic information (i.e., name, work and education, residence, relationships, contacts, profile image); (2) Search by friends and send friend request; (3) Accept friend request; (4) Post content in a public and private way; (5) Posting content on a friend's wall; (6) Interact with posts from others in the news feed; (7) Chat through instant messages with friends; (8) Interacting in events; (9) Interact in groups and (10) Interact with notifications. The main results obtained in this step are presented below. After the inspection steps, the data collected by the experts were analyzed and consolidated so that it was possible to discuss the potential impacts of the features offered on Facebook in the experience of use and sociability.

\subsection{Results}

Through the evaluation, we identified 20 potential problems that violated at least one of the UX and sociability guidelines, which $60 \%$ were classified with severity 3 (i.e., large problem), 30\% with severity 4 (i.e., catastrophic problem), and $10 \%$ with severity 2 (i.e., minor problems). Among the identified potential problems, it is possible to exemplify the difficulties of perceiving and accessing the options for editing and deleting a comment in a publication. As shown in Figure 1, the Facebook interface does not explicitly tell the user that a comment can be edited or deleted after submission. These options are hidden in the interface and, to view them, the user must hover the pointer to the right of the comment so that a button with the image of three dots (ellipsis points) appears. Thus, the way how Facebook provides the options for editing and deleting 
a comment may be a potential problem for senior users that can compromise the social interaction mediated by this online platform. Once, as the options are not explicitly on interface (i.e., they become visible only after dynamic interaction with the system), the user can make limited use or ignore the existence of these options.

As each identified potential problem may be associated with violation of one or more UX and sociability guidelines and the same guideline may be violated by different problems, to conduct the proposed analysis and to present the results in a consolidated manner, we verified how many guidelines were violated and how often these violations occurred in relation to the total number of problems. Figure 2 shows the consolidated results.

From the data presented in Figure 2, it was possible to observe that the guidelines "UX14 - Ease of access to functionalities", "UX4 - Explicit interaction paths" and "UX12 - Visibility and easy access to all existing information" were violated in, at least, $60 \%$ of the problems. In relation to guidelines specifically focused on promoting sociability and collaboration (e.g., S2 - Encouraging empathy and trust among members, S3 - Encouraging reciprocity and S6 - Ensuring protection and privacy), have been mapped to at least $20 \%$ potential identified problems.

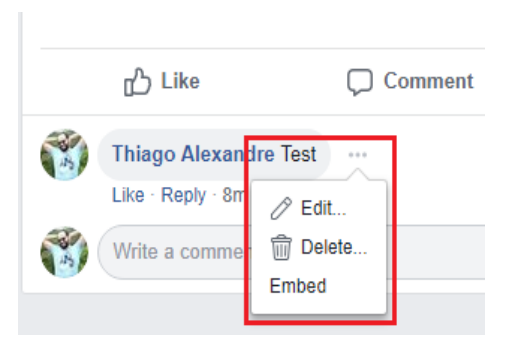

Figure 1. Example of a problem on Facebook interface

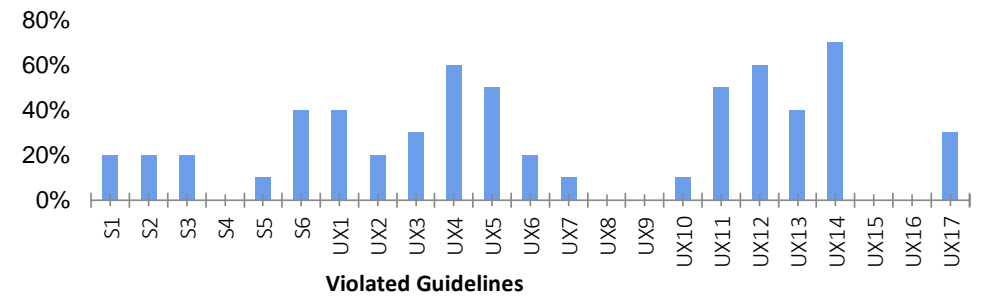

Figure 2. Frequency of violations of UX guidelines and Sociability on Facebook

Although the Facebook offers different resources to promote interaction among its members, considering the profile of elderly users, the violation of the guidelines mentioned above may impair the quality of elderly interaction. Once the Facebook does not explicit the possibilities of interaction in order to facilitate and stimulate the access and use by elderly people, this kind of user can ignore or make limited use of the offered resources. Consequently, Facebook may not be supporting social interaction of elderly users adequately through its interface (Braun, 2013) (Bell et al., 2013).

\section{SOCIABILITY ON FACEBOOK THROUGH ELDERLY USERS' PERSPECTIVE}

In order to investigate how elderly people have been using Facebook, as well as to characterize the perceptions and opinions of them regarding the interaction mediated by this social network, two evaluations were performed in this research: (1) Evaluation in Controlled Environment and (2) Survey with Facebook' users. Next, we present these steps and the results.

\subsection{Evaluation in Controlled Environment}

We performed the evaluation in controlled environment in three stages: preparation, execution and analysis. All steps were conducted by two evaluators. During the preparation phase, we determined the objectives and tasks to be performed during the evaluation. In addition, the profile of the participants was defined, as well as the consent term. Finally, before the execution phase, a pilot test was conducted to verify that all the definitions and generated artifacts were adequate for the evaluation (Prates and Barbosa, 2003).

In the execution stage, the evaluations were performed with each selected participant. During this stage, the following steps were followed for each evaluation session: (1) receipt of the user, (2) presentation of evaluation objectives, (3) obtaining the participant's formal consent, (4) application of the pre-test, (5) observation of tasks performed by the user and (6) interview after test (Prates and Barbosa, 2003)

The following tasks were proposed to the participants: (T1) Create account in Facebook; (T2) Find friends through personal contacts; (T3) Configure profile; (T4) Send a message to friend; (T5) Delete friend; (T6) Post 
in timeline; (T7) Comment on post; (T8) Like Comment, (T9) Edit comment; (T10) Delete comment, and (T11) Access settings menu. During each assessment session, the evaluator noted and recorded: (1) the completion level of each task (i.e., completed without error, completed with error or not completed) and (2) questions and observations made by the participant during the tasks and the final interview.

In total, 17 evaluation sessions were held with different participants over 60 years of age. Among these participants, $60 \%$ declared to be identified with the female gender and $40 \%$ with the male gender. The 17 participants have been using Facebook for at least 6 months. In relation to the weekly frequency of use, all users declared to access the Facebook at least twice a week with the help of a relative. These 17 participants were selected using the saturation sampling technique (Marshall, 1996).

\subsubsection{Results and Discussions}

In relation to the execution of the tasks, the graph of Figure 3 shows the percentage of completion of each task by the participants. From these data, we can see that eight tasks (72\%) were completed with error or were not completed by at least $40 \%$ (seven) of users. In addition, it is possible to highlight that the greatest difficulties of interaction focused on tasks related to creating an account (T1), finding friends (T2) and sending a message to a friend (T4).

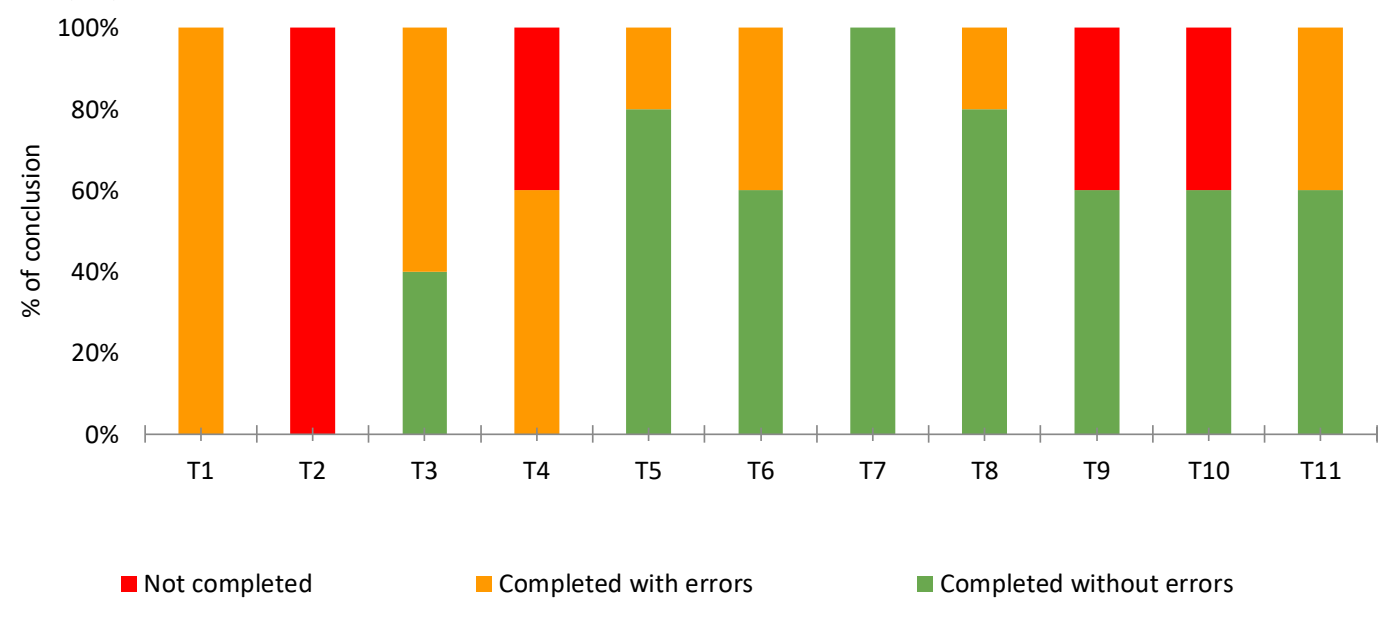

Figure 3. Percentage of completion of Tasks by Elderly Users

Regarding the task to locate and add personal contacts (T2), elderly participants could not locate and invite friends through the contacts registered in their personal emails. Participants in the evaluation sessions indicated that they did not perform this task because they did not understand whether the data requested by Facebook corresponded to (1) access information (i.e., login) of the email account or (2) Facebook itself or (3) because they hoped to find other ways to find friends on the network. To better understand these results, we asked to the participants about their experiences during the tasks execution. One participant P13 said: "I did not execute the task because it must have an easier way to find my friends. I do not have email to locate anyone "[P13].

Since all participants in this research already had a profile on Facebook, the difficulties and problems observed and reported by elderly people have caught the attention of the researchers. Moreover, the participants said that, in addition to the difficulties reported, there were other factors that contributed to the problems experienced during the execution of the tasks (e.g., difficulties to access the functionalities and information).

The results of the completion of the tasks (summarized in Figure 3) as well as the experience and perceptions report by senior people during the interview suggest that elderly users may encounter limitations to initiate the use of features offered by Facebook without the help of other people. These limitations may be associated with the potential problems identified by HCI specialists, especially those related to the difficulty of perception, recognition and use of available resources for interaction. Thus, the reported results suggest that the interface and interaction model of Facebook demands adaptations to enhance the sociability of old users. 


\subsection{Sociability Survey}

The next step of this work was to analyze the perceptions and opinions of elderly users about the resources offered to stimulate social interaction on Facebook. To achieve this goal, an online questionnaire with seven questions was applied. The questions were grouped so that it was possible to identify: (1) the profile of the participants, (2) the participants' perceptions about Facebook's sociability features, and (3) suggestions to enhance the user experience and sociability in this social network. The questionnaire was publicly posted on Facebook on the researchers' personal page, as well as in Facebook communities focused on bringing together elderly people. The survey took place throughout the month of March 2018 and the four authors of this study analyzed the collected data.

The research involved the participation of 121 elderly people who access Facebook at least three times a week and use this service for at least one year. Regarding gender, $78 \%$ of elderly people were identified with the female gender and $22 \%$ with the male gender. The majority $(56 \%)$ reported being in the age group between 60 and 65 years old, while $22 \%$ declared to be between 66 and 70 years old, $17 \%$ between 71 and 75 years old and $5 \%$ in the range between 76 and 80 years old. None of the participants claimed to be over 80 years old.

\subsubsection{Users' Perceptions and Suggestions about the Sociability Features}

Initially, the participants were asked to respond who was responsible for creating their personal accounts on Facebook. Among the participants, 50\% of elderly users stated that friends and family were responsible for this task. On the other hand, $44 \%$ reported having created their own social network accounts and $6 \%$ of elderly people do not remember this information. These data converge with the data collected during the evaluation in controlled environment, once more than half of the participants in this phase also reported that family members created their accounts. These results point to a possible dependence experienced by elderly people during the process of account creation and initialization in the use of Facebook.

Regarding the motivations of using Facebook, the participants could indicate more than one reason. The collected data allowed us to identify that $83 \%$ of elderly people use Facebook to follow the content published on this platform and $78 \%$ access this online network to keep in touch with family and friends. In addition, 33\% are on Facebook to keep old contacts, but also, make new friends, especially of other age groups.

Next, the participants were asked about: (1) what Facebook features they knew and (2) how often they use each known feature. The graph of Figure 4 summarizes the results obtained. It is possible to observe that at least $34 \%$ of participants stated that they did not know, did not use or rarely used all of the features present on Facebook to support social interaction. The limited knowledge and use of these features are an alert, since among these features unknown by elderly users, there are the resources that should be used to promote sociability, such as: (1) maintaining personal information in the profile, (2) find friends, and (3) share publications of interest.

Next, elderly people were asked to indicate suggestions that could contribute to improving the user experience and sociability on Facebook. Thematic analysis (Braun and Clarke, 2006) was used to identify and categorize the suggestions in participants' responses. The graph of Figure 5 summarizes the obtained results. As shown in Figure 5, all participants contributed with suggestions. Among the demands for improvements most cited by at least $70 \%$ of participants, it is possible to highlight: (1) "Ensure that error messages are easy to understand and help the user to take the correct action (suggested by 73\%)"; (2) "Inform clearly what action is being taken by the user and the consequences of each action" (suggested by 77\%); (3) "Facilitate listening and viewing content" (suggested by 70\%), and (4) "Providing ways to help users navigate, find content" (suggested by $84 \%$ ). In addition, it was possible to observe that most of the suggestions are related to: (1) ease of use, (2) feedback, (3) flexibility and (4) stimulation of social interaction in different ways. 
Maintain personal information on the profile Mantain personal preferences on the profile Update personal information privacy on the profile Find a friend through your e-mail address Find a friend through its name Talk to a friend through private chat Send an image on private chat Send a gif on private chat Send stickers on private chat Send an emoji on private chat Post something on a friend's timeline Post a text on your timeline
- Unknown

Unsued

- Rarely used

Erequently used

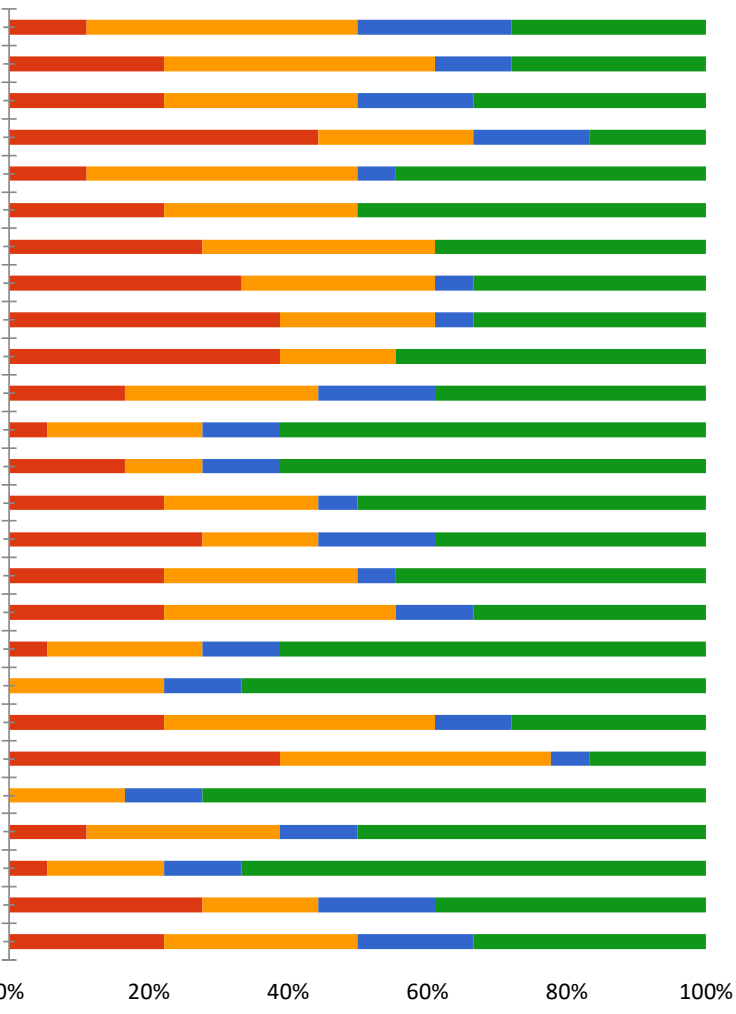

Figure 4. Sociability features of Facebook used by participants

Allow the user to develop their identity online Encourage empathy and trust

Encourage meaningful interaction Mantain easy access to publications

Provide different means of interaction

Ensure member's protection and privacy

Ensure people know how to use existing features Error messages are easy to understand and help the user to perform..

Clearly state what action is being taken by the user and what. Offer multiple paths to perform tasks and inform all available options

Provide explaining texts to any non-textual content Allow interface modification or adaptation to facilitate the use

Facilitate listening and viewing content

Prevent the application from generating any malaise to the user

Provide help navigating and locating content

Make text content readable and understandable

Ensure that user do not need to "guess" what existing feature Ensure that all information is visible, legible, and with easy access Allow the user to get the information they need easily, with no need Ensure that key featutes are easy to access

Make sure that there is a Help option to specify the common Ensure that related information is nearby and the screen is well used

- Can be improved Encourage using the social network

Facebook characteristic/feature unknown

- Unecessary characteristic/feature

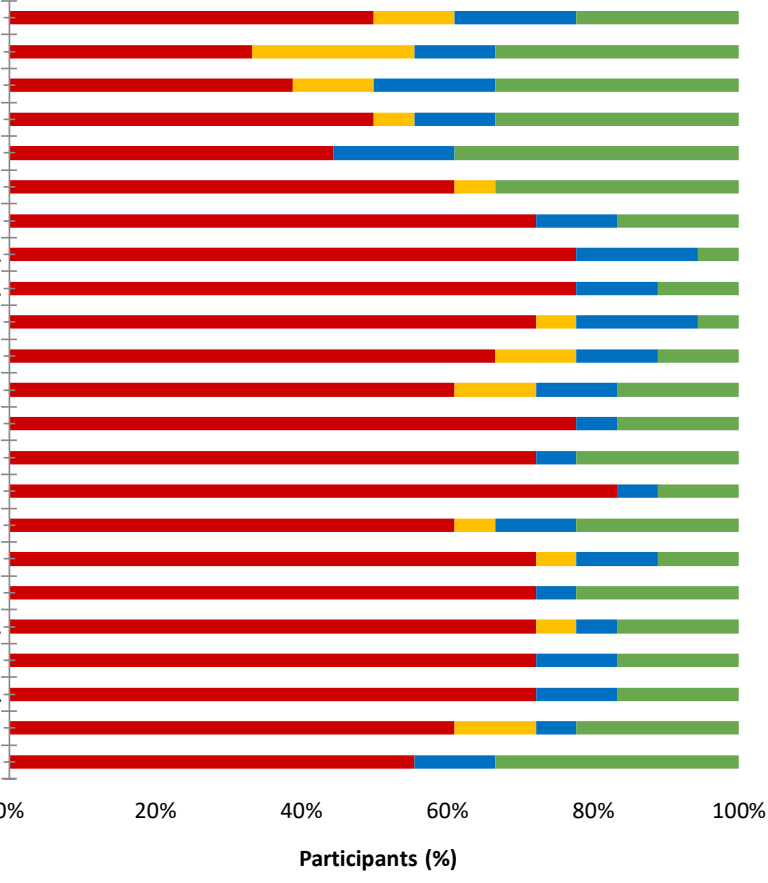

Does not need improvements

Participants (\%)

Figure 5. Sociability features of Facebook that, in the users' view point, can be improved 


\section{DISCUSSION OF RESULTS}

In this research, we investigated whether: "Does the interface and interaction model of Facebook support the social interaction between elderly users?". Initially, the results showed that Facebook offers features to support the sociability of its users, such as (1) sharing and viewing content and (2) diversified interaction with friends through posts, comments and chats. However, considering the profile and needs of elderly users, the HCI experts' evaluation showed that the Facebook violates some UX and sociability guidelines that seek to promote and stimulate social interaction on this type of platform. By violating these guidelines, Facebook may not adequately support the social interaction of senior people through its interface (Braun, 2013) (Bell et al., 2013).

Later, it was possible to show that elderly users may be facing difficulties to recognize and use the resources offered for social interaction on Facebook. For a significant number of participants, this system does not use interface and interaction elements consistent with their expectations and experiences. In addition, the discussed and presented results point to the fact that the problems reported by elderly people during the interaction often affect the autonomy of this audience when using Facebook. Aware of the limited use that has been made of the offered resources, both elderly participants of this research and the HCI specialists presented suggestions for improvements (listed in the graph of Figure 5) that could be incorporated into the interface and interaction model of Facebook so that this network can enhance the sociability of elderly people.

\section{CONCLUSIONS AND FUTURE WORKS}

Taken together, the findings presented and discussed in this paper advance the understandings about UX and sociability in online social networks for elderly public. Specifically, this study indicates how Facebook's interface and interaction model supports and limits sociability for users over 60 years old.

These results contribute to the HCI community may reflect about the importance of addressing the principles of UX and sociability on social software. This study also reinforces the importance of sociability as a strategy to improve the experience and social interaction of elderly people in online social networks. This is a relevant contribution, since elderly population is growing and they can find in social networks a favorable virtual environment to reduce social isolation (Chen and Schulz, 2016). Thus, this paper provides insights and recommendations that are useful for designing online social networks more focused on sociability of elderly people in the future. Furthermore, the adopted methodology might be reproduced in the evaluation of other social medias, considering the UX and social interaction of different interest groups (e.g., teenagers).

As future work, it is possible to suggest the implementation and evaluation of recommendations for the design of online social networks that enhance the social inclusion of elderly people. In addition, other surveys can be conducted to characterize the support of online social networks in the social interaction of different groups of users (e.g., adolescents and people with special needs).

\section{REFERENCES}

Bell, C., Fausset, C., Farmer, S., Nguyen, J., Harley, L. and Fain, W.B., 2013, May. Examining social media use among older adults. In Proceedings of the 24th ACM conference on hypertext and social media (p. 158-163).

Braun, V. and Clarke, V., 2006. Using thematic analysis in psychology. Qualitative research in psychology, p.77-101.

Braun, M.T., 2013. Obstacles to social networking website use among older adults. In Computers in Human Behavior, p.673-680.

Chen, Y.R.R. and Schulz, P.J., 2016. The effect of information communication technology interventions on reducing social isolation in elderly people: a systematic review. In Journal of medical Internet research, 18(1).

Marshall, M.N., 1996. Sampling for qualitative research. Family practice, 13(6), pp.522-526.

Nielsen, J., 1994. Usability inspection methods. In CHI'94, p. 413-414.

Nielsen, J. and Budiu, R. 2015. User Experience for Mobile Applications and Websites. In Design Guidelines, 506p.

Prates, R.O. and Barbosa, S.D.J., 2003. Avaliação de interfaces de usuário-conceitos e métodos. In Jornada de Atualização em Informática do Congresso da Sociedade Brasileira de Computação (Vol. 6, p. 28).

Souza, C.S. de and Preece, J., 2004. A framework for analyzing and understanding online communities. In Interacting with computers, 16(3), p.579-610. 